\title{
Artificial intelligence in arthroplasty
}

\author{
Glen Purnomo $0^{1,2^{*}} \mathbb{D}$, Seng-Jin Yeo ${ }^{2,3}$ and Ming Han Lincoln Liow ${ }^{2,3}$
}

\begin{abstract}
Artificial intelligence (Al) is altering the world of medicine. Given the rapid advances in technology, computers are now able to learn and improve, imitating humanoid cognitive function. Al applications currently exist in various medical specialties, some of which are already in clinical use. This review presents the potential uses and limitations of Al in arthroplasty to provide a better understanding of the existing technology and future direction of this field.

Recent literature demonstrates that the utilization of Al in the field of arthroplasty has the potential to improve patient care through better diagnosis, screening, planning, monitoring, and prediction. The implementation of Al technology will enable arthroplasty surgeons to provide patient-specific management in clinical decision making, preoperative health optimization, resource allocation, decision support, and early intervention. While this technology presents a variety of exciting opportunities, it also has several limitations and challenges that need to be overcome to ensure its safety and effectiveness.
\end{abstract}

Keywords: Artificial intelligence, Deep learning, Machine learning, Arthroplasty, Joint replacement

\section{Background}

Artificial Intelligence (AI) is a subfield of computer science that uses computers to emulate human cognitive functions and carry out tasks that can match or exceed human performance $[1,2]$. Traditionally, specific computer codes were required to explicitly instruct machines how to process data and make decisions. Through Machine Learning (ML) algorithms, computers can learn and improve from experience without exact instructions by using large sets of data inputs and outputs to recognize repetitive patterns. This subset of AI emphasizes the learning aspect of computer intelligence to create autonomous resolutions $[1,3,4]$.

Deep Learning (DL) is a more advanced and complex form of ML that mimics the neuronal connections of the brain by creating an Artificial Neural Network (ANN) $[1,2]$. This algorithm can learn from unstructured and unlabeled inputs without supervision and segregate data input from low relevance variables for the prediction

\footnotetext{
*Correspondence: glen.purnomo@yahoo.com

1 St. Vincentius a Paulo Catholic Hospital, Surabaya, Indonesia

Full list of author information is available at the end of the article
}

of interest. Deep Neural Network (DNN), which contains multiple hierarchical levels of $\mathrm{ANN}$, is required to improve data predictions and allow the development of models without explicitly programmed directions [1-3]. Convolutional Neural Network $(\mathrm{CNN})$ is the other form of DL which is used for computer vision tasks including medical image analysis [5].

The application of AI has expanded prominently in the medical field due to advances in computing power, learning algorithms, data storage, and the availability of large-high-quality data sourced from electronic medical records and wearable health trackers $[1,2]$. Although its adoption is still in early phases, AI has been extensively used across many fields in medicine such as radiology [6, 7], cardiology [8-11], dermatology [12-15], ophthalmology $[16,17]$, neurology $[18,19]$, oncology $[20,21]$, gastroenterology [22, 23], and respiratory medicine [24]. Some examples of clinical applications that have been approved by the US Food and Drug Administration (FDA) include Arterys for cardiac magnetic resonance image analysis, $I d x$ for detection of diabetic retinopathy, and MammoScreen for breast cancer screening $[25,26]$. In fact, the number of $\mathrm{AI} / \mathrm{ML}$-based medical devices approved original author(s) and the source, provide a link to the Creative Commons licence, and indicate if changes were made. The images or other third party material in this article are included in the article's Creative Commons licence, unless indicated otherwise in a credit line to the material. If material is not included in the article's Creative Commons licence and your intended use is not permitted by statutory regulation or exceeds the permitted use, you will need to obtain permission directly from the copyright holder. To view a copy of this licence, visit http://creativecommons.org/licenses/by/4.0/. 
by the FDA and Conformité Européenne (CE) mark in Europe increased significantly from 9 and 13 in 2015 to 77 and 100 in 2019, respectively [26].

In the field of orthopedics, the influence of AI has also grown rapidly for the past two decades [4]. Currently, this technology has the potential to be utilized in various areas such as image-recognition diagnostics, surgical risk prediction, generating patient-specific payment models, augmenting clinical decision making, and outcome prediction [2]. Thus, AI enables surgeons to select the ideal patient for the best surgical outcomes, develop a patientspecific surgical plan, and prepare for patients who are at high risk of complications [27].

This article reviews the current applications of AI in the field of arthroplasty and the evidence supporting its utilization in the clinical setting.

\section{Diagnostic tool}

Computer-aided diagnosis could be utilized to help physicians make decisions because of its objective nature and a high degree of accuracy. $\mathrm{CNN}$ has demonstrated breakthroughs in a variety of general image recognition applications. The accuracy of these applications is at near-human levels and has the potential to outperform human experts in the future [28]. These models can be used to aid physicians to diagnose osteoarthritis, assess the severity of osteoarthritis, or even detect osteoarthritis in presymptomatic individuals and predict the need for joint arthroplasty [28-34].

Diagnosis and progression of osteoarthritis (OA) based on knee radiograph can be made by CNN. Several algorithms have been proposed to detect knee OA based on knee radiographs. By using plain radiographs from the OsteoArthritis Initiative database, Brahim et al demonstrated the ability of their method to detect OA, even at an early stage [35]. Another DL model developed by Xue et al was able to generate a diagnosis of OA automatically using a radiograph without human involvement. This algorithm could accurately identify radiograph features that are associated with hip OA such as joint space narrowing and osteophytes, allowing it to function like a radiologist with 10 years of experience [29]. Both Antony et al and Tiulpin et al presented algorithms that were able to automatically diagnose and classify the severity of knee OA from plain radiographs $[28,32]$. Most recently, the model proposed by Leung et al not only detected knee OA but also predicted the development of OA which led to eventual total knee arthroplasty (TKA) within 9 years [31].

In a different application, early diagnosis of $\mathrm{OA}$ in presymptomatic individuals may allow initiation of diseasemodifying therapies to modify the course of the disease. Kundu et al demonstrated the use of AI to detect OA
3 years before the individuals became symptomatic. They used 3D Transport-Based Morphometry to identify water distribution patterns in cartilage tissue that is captured by MRI [30]. In addition, Hirvasniemi et al used ML to predict incidents of radiographic hip OA or TKA over 10 years. They utilized bone texture analysis of proximal femur and acetabulum from plain pelvic radiographs to predict incidents of radiographic hip OA [33].

The recent technological advances in AI have proven that image recognition applications can detect prosthetic loosening. This is particularly useful as aseptic implant loosening remains one of the main causes of failure in arthroplasty. Shah et al showed that loosening of implants could be detected on plain radiographs by ML with an accuracy of $95.6 \%$. This progress could assist surgeons in diagnosing the condition without the need for expensive imaging modalities such as fluorodeoxyglucose-positron emission tomography (FDG-PET scans), bone scans, magnetic resonance imaging (MRI), and arthrograms, which have not been demonstrated to significantly improve diagnostic accuracy [36].

\section{Patient-specific payment}

The use of AI to preoperatively predict the length of stay in the hospital (LOS) and payments for patients undergoing arthroplasty based on patient-specific factors can help optimize value-based care and enable the development of a patient-specific pricing benchmark for governments and insurance companies [37-39]. By accurately predicting the LOS of patients, decision making and inpatient bed assignment can be simplified so that medical resources can be allocated to the maximum [40]. Doctors can also develop recovery plans quickly and provide better services as they can adjust patient expectations and provide early interventions [38]. High-cost predictions allow hospitals to channel additional resources to at-risk patients, to prevent anticipated complications and reduce overall costs [37].

Several studies have demonstrated the ability of ML to predict LOS and hospitalization costs for both TKA and total hip arthroplasty (THA) [37, 39, 41, 42]. ML is proven to provide predictions with fair to excellent construct validity, reliability, and responsiveness before primary TKA and THA [39, 41, 42].

Li et al conducted a study to compare the accuracy of the ML model with two logistic regression models in predicting LOS of patients undergoing primary unilateral TKA. They found that the ML algorithm had better accuracy in predicting LOS [38]. Another similar study has also demonstrated better predictive performance of their ML models compared to conventional methods, including logistic regression [40]. 


\section{Preoperative evaluation}

AI can be used during preoperative screening to identify high-risk patients thereby allowing doctors to prepare preventive measures. Jo et al conducted the first study on the use of ML to predict the transfusion risk after TKA while Karhade et al performed the first investigation into the use of ML in predicting the risk of having prolonged postoperative opioid prescriptions in patients undergoing THA. Both studies have been validated and showed good performance [43, 44].

Novel use of AI has been proposed for the preoperative evaluation of revision arthroplasty. While accurate identification of the manufacturer and type of implant is required for preoperative planning of revision arthroplasty, it is estimated that surgeons are unable to recognize the implant preoperatively and intraoperatively in about 10 and $2 \%$ of cases, respectively. Inability to identify implants can lead to unpreparedness which may contribute to increased surgical time, perioperative morbidity, and overall healthcare costs [45-47]. Deep learning has been shown its ability to recognize implant manufacturer and design in hip and knee arthroplasty [45-49].

A recent study by Karnuta et al showed that their ML algorithm was able to distinguish between 9 unique knee arthroplasty implants including TKA, Unicondylar Knee Arthroplasty, and Distal Femoral Replacement from four leading manufacturers with 99\% accuracy simply by evaluating anteroposterior (AP) radiographs [46]. In hip arthroplasty, deep learning algorithms are also capable of recognizing THA implants based on AP radiograph. Although the classification is based solely on identifying the design characteristics of the femoral stem, studies have found the results to be very accurate, even reaching an accuracy rate of 99.6 to $100 \%$ [45, 47, $49,50]$.

Jodeiri et al estimated Pelvic Sagittal Inclination (PSI) from preoperative plain radiographs to augment the position of the acetabular component, eliminating the need for a Computed Tomography (CT) scan. PSI is increasingly being recognized to play a crucial role in acetabular component positioning as the optimal anteversion and inclination angle of the acetabular cup can reduce the risk of dislocation or impingement after THA. This study demonstrated encouraging results, with an accuracy rate of $80 \%$. Future development of this model will enable the recognition of individual dynamic changes of PSI to enable patient-specific placement of the acetabular component in patients undergoing THA [51].

The application of these novelties during preoperative evaluation has the potential to assist surgeons in making clinical decisions, providing patient-specific planning, and improving outcomes.

\section{Outcome prediction}

The potential ability of ML to predict patient outcomes after arthroplasty has been demonstrated by several authors [52-56]. Prediction of outcomes may facilitate the shared decision-making process between orthopedic surgeons and patients, particularly to decide whether the procedures will meet the patient's expectations [53-56]. Furthermore, early identification of patients at risk of not having significant changes in postoperative PatientReported Outcome Measures (PROMs) may necessitate closer postoperative patient follow-up and optimize decision support before surgery [52].

Several studies focused on achieving minimal clinically important difference (MCID) after surgery, which refers to the improvement of PROMs necessary for a patient to consider the intervention beneficial or meaningful. Supervised machine learning algorithms developed by Fontana et al were shown to have fair to good ability to predict 2-year postsurgical MCID for general and jointspecific health PROMs [52]. Recent studies by Kunze et $a l$ and Harris et al also found that ML was able to predict the MCID of patients undergoing THA and TKA, respectively, so that it could help in optimizing preoperative health, improving patient selection, education, and satisfaction $[55,56]$.

Another outcome measure used to evaluate the efficacy and value of the intervention is postoperative patient satisfaction [54, 57]. Farooq et al found that compared to statistical models, ML algorithm had greater accuracy in predicting satisfaction after TKA [57]. Another study conducted by Kunze et al in patients undergoing TKA also showed that ML had good discriminatory capacity and superiority over standard logistic regression to identify patients at greatest risk for dissatisfaction. Accurate prediction of patient dissatisfaction following primary TKA may allow for preoperative health optimization and improved patient-doctor discussions [54].

Shohat et al. used ML to assist in treatment decision making of acute prosthetic joint infection (PJI). Debridement, antibiotics, and implant retention (DAIR) procedures have low morbidity with unpredictable results and variable failure rates. The authors developed an algorithm that could accurately predict the success of DAIR based on patient's clinical presentations, comorbidities, physical examination, and laboratory results [58].

Shat et al did a retrospective cohort study of 89,986 patients undergoing primary THA. They developed an ML algorithm and compared their model with logistic regression and standard benchmark ML models in predicting major complications after THA, including infection, venous thromboembolism, cardiac complication, and pulmonary complication. Their model showed superior risk prediction compared to logistic regression. The 
accurate prognostic information obtained by this algorithm may facilitate decision making before surgery and augment postoperative clinical care [59].

\section{Postoperative evaluation and monitoring}

Newer technology targeted at improving rehabilitation following THR and TKR provides a more objective outcome measure as compared to traditional patientreported activity questionnaires. Wearable devices can record the movement profile of patients postoperatively, which can be used to assess their functional recovery $[60$, 61]. Continuous data from the device can be analyzed by ML algorithms which can lead to reliable measurement of functional outcomes after arthroplasty [62].

Ramkumar et al analyzed perioperative data collected by patient smartphones and wearable knee devices of patients undergoing TKA. Analysis of the data taken pre- and post-surgery including mobility (steps per day), range of motion (maximum knee flexion), home exercise compliance, opioid consumption, and PROMs, demonstrated that the remote patient monitoring enabled the authors to evaluate the mobility and rehabilitation compliance of patients after TKA. By analyzing data collected through advances in technology with ML algorithms, they proposed that these applications can help surgeons to identify potential causes of unfavorable outcomes [62].

Polus et al did a prospective study investigating the recovery of 72 patients undergoing primary THA for end-stage OA. All patients were instrumented with a wearable sensor system. This study showed that ML could predict the fall risk in post-THA patients by collecting objective functional data using wearable devices. They succeeded in predicting the risk of fall at 6 weeks after surgery with a high level of accuracy. By grouping high and low fall risk patients, fall prevention measures can be enhanced for the high-risk group, whilst an accelerated recovery program can be implemented for the low-risk group [63].

As another example, Rouzrokh et al used DL to automatically measure the acetabular component angles on postoperative radiographs. After building their algorithm based on two cohorts of 600 AP pelvis and 600 crosstable lateral hip postoperative radiographs, they found that their model was highly accurate so that it can be utilized not only in research settings but also in clinical settings [64].

\section{Al for surgical robotics}

Robotic-assisted surgery enhances the surgeon's ability to perform more precise and accurate procedures more consistently with more patient-specific plans [65]. Many surgical robotic platforms have emerged in the last few decades. Despite progress, current robotic platforms are incapable of performing autonomous tasks and making cognitive decisions similar to those of humans [66]. AI may improve the ability of the surgical robotic system to perceive complex in vivo environments, conduct decision making, predict, and perform desired tasks with increased precision, safety, and efficiency, either under or without supervision from human control $[67,68]$.

The application of AI in robotic surgeries may reduce human errors and operative times [69]. Future surgical robots are expected to be able to apprehend and understand complex environments, perform real-time decision making, and perform desired tasks with increased precision, safety, and efficiency [68]. Recent study by Li et al used $\mathrm{AI}$ for the first time to guide the 3D reconstruction of CT data of lower limbs for facilitating robotic-assisted TKA. They used CT data of 200 lower limbs for AI-based 3D model construction and CT data of 20 lower limbs for verification. The result showed that the performance of AI-guided 3D reconstruction for robotic-assisted TKA was similar to that of the operator-based approach [70].

\section{Clinical decision making and future directions}

Advances in technology and the use of AI provide opportunities to provide data-driven, high-performance medicine that can rapidly improve the field of arthroplasty [71]. Leveraging its potential to handle and optimize highly complex datasets, a future where the positive impact of this technology in healthcare is already visible [72]. Due to the ability to process large amounts of complex data to guide and predict outcomes, AI platforms have the potential to provide decision support to doctors, patients, and insurance companies [27, 72].

Arthroplasty surgeons will be able to select ideal patients for surgery, create patient-specific surgical plans, predict clinical outcomes and implant survival, and identify patients at high risk of complications [27]. In addition, the potential of AI to forecast treatment episodes offers unique predictive possibilities for generating tiered bundle pay models specific to patient complexity before arthroplasty procedures, enabling fair arbitration between surgeons, hospitals, and insurance companies [41]. AI's ability to predict outcomes will facilitate arthroplasty surgeons in discussing possible surgery outcomes, making optimal joint decisions with patients before surgery, and prioritizing resources for postoperative monitoring [52].

Advanced algorithms offer an avenue to learn and adapt to different datasets including those relating to a patient's physical and psychosocial health and well-being from different populations and practices. Highly sophisticated analysis of a wide range of data is capable of generating impactful metrics that can be used to aid in better decision-making processes [73]. Jayakumar et al conducted a 
randomized clinical trial of 129 patients with knee pain associated with OA. They demonstrated statistically significant improvement in decision quality, level of shared decision making, patient satisfaction, and functional outcomes in patients using an AI-enabled decision aid. The results of this study suggest that AI-enabled patient decision assistance can provide a personalized, data-driven approach and improve shared decision making in the management of knee osteoarthritis [74].

Given the rapid technological advances, the widespread application of AI in the field of arthroplasty is expected to provide personalized health care by improving diagnosis, clinical decision making, patient care, and outcomes for specific patients.

\section{Al limitations and challenges}

Apart from various interesting and seemingly promising applications, AI also has its limitations. The development of ML algorithms requires large amounts of data. ML developed using data from one setting cannot be used immediately by other practice settings in other locations because the training data may not representative of the population [75]. Algorithms with non-generalized data can lead to bias, possibly providing inaccurate recommendations for minority subgroups for which training data are less inclusive [76, 77]. To prevent algorithmic bias, ML should be designed according to the global community. In addition, clinical validation must be carried out using a representative population of the area where this algorithm will be used [77].

Additional local training data may be required for algorithm adaptation in new populations [75, 77]. Hospitals or clinics with too little data will face problems training the algorithm optimally so that sharing data with each other is necessary to achieve successful adoption of $\mathrm{AI}$ in healthcare at scale [76]. In data sharing, privacy and data protection issues can be a problem. AI developers must protect personal information and any other information beyond the use of a doctor-patient relationship that may harm the patient, such as the impact on health or other insurance premiums, job opportunities, or even personal relationships [76]. Moreover, there are also unique challenges and risks associated with cyber security threats $[76,78]$.

Transparency and trust are other issues. A lack of transparency in AI makes accountability and liability problematic [76, 79]. Some ML algorithms have a blackbox phenomenon in which a logical explanation of how the output is generated is unknown [80]. The inability to explain why and how an algorithm derives certain decisions makes implementing AI difficult [77]. Explainable and interpretable algorithms are necessary not only to detect biases but also to facilitate transparent and trustworthy AI systems [76, 77].

\section{Conclusion}

The adoption of AI in healthcare is inevitable. Currently, many studies are demonstrating the use of $\mathrm{AI}$ in various fields of arthroplasty. The application of ML in clinical practice will allow physicians to improve clinical decision making, anticipate problems, allocate resources, and provide personalized early intervention for each patient. AI has the potential to increase surgeon effectiveness and reduce human errors. Shortly, this technology will surely help arthroplasty surgeons in various ways to improve patient outcomes. While presenting a variety of exciting opportunities, the application of AI is not without limitations, making the adoption of this technology into clinical settings problematic. These challenges need to be addressed to ensure the safe and effective use of this technology.

\section{Abbreviations \\ Al: Artificial intelligence; ANN: Artificial Neural Network; AP: Anteroposterior; CE: Conformité Européenne; CNN: Convolutional Neural Network; CT: Com- puted Tomography; DAIR: Debridement, antibiotics, and implant retention; DL: Deep Learning; DNN: Deep Neural Network; FDA: US Food and Drug Admin- istration; FDG-PET: Fluorodeoxyglucose-positron emission tomography; LOS: Length of stay in the hospital; MCID: Minimal clinically important difference; ML: Machine Learning; MRI: Magnetic resonance imaging; OA: Osteoarthritis; PJl: Prosthetic joint infection; PROMs: Patient-Reported Outcome Measures; PSI: Pelvic sagittal inclination; THA: Total hip arthroplasty; TKA: Total knee arthroplasty.}

\section{Acknowledgements}

Not applicable.

\section{Authors' contributions}

GP was the main contributor in writing the manuscript, SJY reviewed and provided inputs on the manuscript, MHLL conceptualized and reviewed the manuscript. All authors read and approved the final manuscript.

\section{Funding}

The authors received no financial support for the research, authorship, and/or publication of this article.

\section{Availability of data and materials}

Not applicable.

\section{Declarations}

Ethics approval and consent to participate

Not applicable.

Consent for publication

Not applicable.

\section{Competing interests}

The authors declare that they have no competing interests.

Author details

${ }^{1}$ St. Vincentius a Paulo Catholic Hospital, Surabaya, Indonesia. ${ }^{2}$ Adult Reconstruction Service, Department of Orthopaedic Surgery, Singapore General 
Hospital, Singapore, Singapore. ${ }^{3}$ Duke-NUS Medical School, Singapore, Singapore

\section{Received: 14 June 2021 Accepted: 31 August 2021}

Published online: 02 November 2021

\section{References}

1. Ahuja AS. The impact of artificial intelligence in medicine on the future role of the physician. PeerJ. 2019;7:e7702. https://doi.org/10.7717/peerj. 7702. Published 2019 Oct 4.

2. Myers TG, Ramkumar PN, Ricciardi BF, Urish KL, Kipper J, Ketonis C. Artificial intelligence and orthopaedics. J Bone Jt Surg. 2020;102(9):830-40.

3. Helm JM, Swiergosz AM, Haeberle HS, Karnuta JM, Schaffer JL, Krebs VE, et al. Machine learning and artificial intelligence: definitions, applications, and future directions. Curr Rev Musculoskelet Med. 2020;13(1):69-76.

4. Ramkumar PN, Kunze KN, Haeberle HS, Karnuta JM, Luu BC, Nwachukwu $\mathrm{BU}$, et al. Clinical and research medical applications of artificial intelligence. Arthrosc J Arthrosc Relat Surg Off Publ Arthrosc Assoc North Am Int Arthrosc Assoc. 2021;37(5):1694-7.

5. Yamashita R, Nishio M, Do RKG, Togashi K. Convolutional neural networks: an overview and application in radiology. Insights Imaging. 2018:9(4):611-29.

6. RezazadeMehrizi MH, van Ooijen P, Homan M. Applications of artificial intelligence (Al) in diagnostic radiology: a technography study. Eur Radiol. 2021;31(4):1805-11

7. Mun SK, Wong KH, Lo SCB, Li Y, Bayarsaikhan S. Artificial intelligence for the future radiology diagnostic service. Front Mol Biosci. 2020;7:614258.

8. Lopez-Jimenez F, Attia Z, Arruda-Olson AM, Carter R, Chareonthaitawee $\mathrm{P}$, Jouni $\mathrm{H}$, et al. Artificial intelligence in cardiology: present and future. Mayo Clin Proc. 2020;95(5):1015-39.

9. Itchhaporia D. Artificial intelligence in cardiology. Trends in cardiovascular medicine. Elsevier Inc.; 2020. https://doi.org/10.1016/j.tcm.2020.11.007.

10. Hannun AY, Rajpurkar P, Haghpanahi M, Tison GH, Bourn C, Turakhia MP, et al. Cardiologist-level arrhythmia detection and classification in ambulatory electrocardiograms using a deep neural network. Nat Med. 2019;25(1):65-9.

11. Johnson KW, Torres Soto J, Glicksberg BS, Shameer K, Miotto R, Ali M, et al. Artificial intelligence in cardiology. J Am Coll Cardiol. 2018;71:2668-79. Elsevier USA.

12. Esteva A, Kuprel B, Novoa RA, Ko J, Swetter SM, Blau HM, et al. Dermatologist-level classification of skin cancer with deep neural networks. Nature. 2017:542(7639):115-8.

13. Haenssle HA, Fink C, Toberer F, Winkler J, Stolz W, Deinlein T, et al. Man against machine reloaded: performance of a market-approved convolutional neural network in classifying a broad spectrum of skin lesions in comparison with 96 dermatologists working under less artificial conditions. Ann Oncol Off J Eur Soc Med Oncol. 2020;31(1):137-43.

14. Haenssle HA, Fink C, Schneiderbauer R, Toberer F, Buhl T, Blum A, et al. Man against machine: diagnostic performance of a deep learning convolutional neural network for dermoscopic melanoma recognition in comparison to 58 dermatologists. Ann Oncol. 2018;29(8):1836-42.

15. Brinker TJ, Hekler A, Enk AH, Klode J, Hauschild A, Berking C, et al. Deep learning outperformed 136 of 157 dermatologists in a head-tohead dermoscopic melanoma image classification task. Eur J Cancer. 2019:113:47-54

16. Gargeya R, Leng T. Automated identification of diabetic retinopathy using deep learning. Ophthalmology. 2017:124(7):962-9.

17. Gulshan V, Peng L, Coram M, Stumpe MC, Wu D, Narayanaswamy A, et al. Development and validation of a deep learning algorithm for detection of diabetic retinopathy in retinal fundus photographs. JAMA. 2016;316(22):2402-10.

18. Pedersen M, Verspoor $K$, Jenkinson M, Law M, Abbott DF, Jackson GD. Artificial intelligence for clinical decision support in neurology. Brain Commun. 2020;2(2):fcaa096.

19. Mathotaarachchi S, Pascoal TA, Shin M, Benedet AL, Kang MS, Beaudry T, et al. Identifying incipient dementia individuals using machine learning and amyloid imaging. Neurobiol Aging. 2017:59:80-90.

20. Sobhani F, Robinson R, Hamidinekoo A, Roxanis I, Somaiah N, Yuan Y. Artificial intelligence and digital pathology: opportunities and implications for immuno-oncology. Biochim Biophys Acta Rev Cancer. 2021;1875:188520. Elsevier B.V.

21. Elkhader J, Elemento O. Artificial intelligence in oncology: from bench to clinic. Semin Cancer Biol. 2021. https://doi.org/10.1016/j.semcancer.2021. 04.013. Academic Press.

22. Parasa S, Wallace M, Bagci U, Antonino M, Berzin T, Byrne M, et al. Proceedings from the first global artificial intelligence in gastroenterology and endoscopy Summit. Gastrointest Endosc. 2020;92(4):938-945.e1.

23. Parasher G, Wong M, Rawat M. Evolving role of artificial intelligence in gastrointestinal endoscopy. World J Gastroenterol. 2020;26(46):7287-98.

24. Kaplan A, Cao H, FitzGerald JM, lannotti N, Yang E, Kocks JWH, et al. Artificial intelligence/machine learning in respiratory medicine and potential role in asthma and COPD diagnosis. J Allergy Clin Immunol Pract. 2021;9(6):2255-61.

25. Benjamens S, Dhunnoo P, Meskó B. The state of artificial intelligencebased FDA-approved medical devices and algorithms: an online database. NPJ Digit Med. 2020;3(1):1-8. https://doi.org/10.1038/ s41746-020-00324-0.

26. Muehlematter UJ, Daniore P, Vokinger KN. Approval of artificial intelligence and machine learning-based medical devices in the USA and Europe (2015-20): a comparative analysis. Lancet Digit Health. 2021;3(3):e195-203.

27. Magan AA, Kayani B, Chang JS, Roussot M, Moriarty P, Haddad FS. Artificial intelligence and surgical innovation: lower limb arthroplasty. Br J Hosp Med. 2020;81(10):1-7.

28. Tiulpin A, Thevenot J, Rahtu E, Lehenkari P, Saarakkala S. Automatic knee osteoarthritis diagnosis from plain radiographs: a deep learning-based approach. Sci Rep. 2018;8(1):1-10.

29. Xue Y, Zhang R, Deng Y, Chen K, Jiang T. A preliminary examination of the diagnostic value of deep learning in hip osteoarthritis. PLoS One. 2017;12(6):1-9

30. Kundu S, Ashinsky BG, Bouhrara M, Dam EB, Demehri S, Shifat-E-Rabbi $M$, et al. Enabling early detection of osteoarthritis from presymptomatic cartilage texture maps via transport-based learning. Proc Natl Acad Sci U S A. 2020;117(40):24709-19.

31. Leung K, Zhang B, Tan J, Shen Y, Geras KJ, Babb JS, et al. Prediction of total knee replacement and diagnosis of osteoarthritis by using deep learning on knee radiographs: data from the osteoarthritis initiative. Radiology. 2020;296(3):584-93.

32. Antony J, McGuinness K, O'Connor N, Moran K. Quantifying radiographic knee osteoarthritis severity using deep convolutional neural networks. In: 2016 23rd Int Conf Pattern Recognit. 2016. p. 1195-200.

33. Hirvasniemi J, Gielis WP, Arbabi S, Agricola R, van Spil WE, Arbabi V, et al. Bone texture analysis for prediction of incident radiographic hip osteoarthritis using machine learning: data from the Cohort Hip and Cohort Knee (CHECK) study. Osteoarthr Cartil. 2019:27(6):906-14.

34. Lim J, Kim J, Cheon S. A deep neural network-based method for early detection of osteoarthritis using statistical data. Int J Environ Res Public Health. 2019;16(7):1281

35. Brahim A, Jennane $R$, Riad $R$, Janvier $T$, Khedher $L$, Toumi $H$, et al. A decision support tool for early detection of knee OsteoArthritis using $X$-ray imaging and machine learning: data from the OsteoArthritis Initiative. Comput Med Imaging Graph Off J Comput Med Imaging Soc. 2019;73:11-8.

36. Shah RF, Bini SA, Martinez AM, Pedoia V, Vail TP. Incremental inputs improve the automated detection of implant loosening using machinelearning algorithms. Bone Jt J. 2020;102-B(6):101-6.

37. Karnuta JM, Navarro SM, Haeberle HS, Helm JM, Kamath AF, Schaffer JL, et al. Predicting inpatient payments prior to lower extremity arthroplasty using deep learning: which model architecture is best? J Arthroplasty. 2019;34(10):2235-2241.e1.

38. Li H, Jiao J, Zhang S, Tang H, Qu X, Yue B. Construction and comparison of predictive models for length of stay after total knee arthroplasty: regression model and machine learning analysis based on 1,826 cases in a Single Singapore center. J Knee Surg. 2020. https://doi.org/10.1055/s0040-1710573.

39. Navarro SM, Wang EY, Haeberle HS, Mont MA, Krebs VE, Patterson BM, et al. Machine learning and primary total knee arthroplasty: patient forecasting for a patient-specific payment model. J Arthroplasty. 2018;33(12):3617-23. 
40. Han C, Liu J, Wu Y, Chong Y, Chai X, Weng X. To predict the length of hospital stay after total knee arthroplasty in an orthopedic center in China: the use of machine learning algorithms. Front Surg. 2021;8:606038.

41. Ramkumar PN, Navarro SM, Haeberle HS, Karnuta JM, Mont MA, lannotti JP, et al. Development and validation of a machine learning algorithm after primary total hip arthroplasty: applications to length of stay and payment models. J Arthroplasty. 2019;34(4):632-7.

42. Ramkumar PN, Karnuta JM, Navarro SM, Haeberle HS, Scuderi GR, Mont $M A$, et al. Deep learning preoperatively predicts value metrics for primary total knee arthroplasty: development and validation of an artificial neural network model. J Arthroplasty. 2019;34(10):2220-2227.e1.

43. Jo C, Ko S, Shin WC, Han H-S, Lee MC, Ko T, et al. Transfusion after total knee arthroplasty can be predicted using the machine learning algorithm. Knee Surg Sports Traumatol Arthrosc. 2020;28(6):1757-64.

44. Karhade AV, Schwab JH, Bedair HS. Development of machine learning algorithms for prediction of sustained postoperative opioid prescriptions after total hip arthroplasty. J Arthroplasty. 2019;34(10):2272-2277.e1.

45. Borjali A, Chen AF, Muratoglu OK, Morid MA, Varadarajan KM. Detecting total hip replacement prosthesis design on plain radiographs using deep convolutional neural network. J Orthop Res. 2020:38(7):1465-71.

46. Karnuta JM, Luu BC, Roth AL, Haeberle HS, Chen AF, lorio R, et al. Artificial intelligence to identify arthroplasty implants from radiographs of the knee. J Arthroplasty. 2021;36(3):935-40.

47. Karnuta JM, Haeberle HS, Luu BC, Roth AL, Molloy RM, Nystrom LM, et al. Artificial intelligence to identify arthroplasty implants from radiographs of the hip. J Arthroplasty. 2021;36(75):S290-S294.e1.

48. Yi PH, Wei J, Kim TK, Sair HI, Hui FK, Hager GD, et al. Automated detection \& classification of knee arthroplasty using deep learning. Knee. 2020;27(2):535-42.

49. Murphy M, Killen C, Burnham R, Sarvari F, Wu K, Brown N. Artificial intelligence accurately identifies total hip arthroplasty implants: a tool for revision surgery. Hip Int J Clin Exp Res Hip Pathol Ther. 2021;1120700020987526. https://doi.org/10.1177/1120700020987526.

50. Kang Y-J, Yoo J-I, Cha Y-H, Park CH, Kim J-T. Machine learning-based identification of hip arthroplasty designs. J Orthop Transl. 2020;21:13-7.

51. Jodeiri A, Zoroofi RA, Hiasa Y, Takao M, Sugano N, Sato Y, et al. Fully automatic estimation of pelvic sagittal inclination from anterior-posterior radiography image using deep learning framework. Comput Methods Programs Biomed. 2020;184:105282.

52. Fontana MA, Lyman S, Sarker GK, Padgett DE, MacLean CH. Can machine learning algorithms predict which patients will achieve minimally clinically important differences from total joint arthroplasty? Clin Orthop Relat Res. 2019;477(6):1267-79.

53. Huber M, Kurz C, Leidl R. Predicting patient-reported outcomes following hip and knee replacement surgery using supervised machine learning. BMC Med Inform Decis Mak. 2019;19(1):1-13.

54. Kunze KN, Polce EM, Sadauskas AJ, Levine BR. Development of machine learning algorithms to predict patient dissatisfaction after primary total knee arthroplasty. J Arthroplasty. 2020;35(11):3117-22.

55. Harris AHS, Kuo AC, Bowe TR, Manfredi L, Lalani NF, Giori NJ. Can machine learning methods produce accurate and easy-to-use preoperative prediction models of one-year improvements in pain and functioning after knee arthroplasty? J Arthroplasty. 2021;36(1):112-117.e6.

56. Kunze KN, Karhade AV, Sadauskas AJ, Schwab JH, Levine BR. Development of machine learning algorithms to predict clinically meaningful improvement for the patient-reported health state after total hip arthroplasty. J Arthroplasty. 2020;35(8):2119-23.

57. Farooq H, Deckard ER, Ziemba-Davis M, Madsen A, Meneghini RM. Predictors of patient satisfaction following primary total knee arthroplasty: results from a traditional statistical model and a machine learning algorithm. J Arthroplasty. 2020;35(11):3123-30.

58. Shohat N, Goswami K, Tan TL, Yayac M, Soriano A, Sousa R, et al. 2020 Frank Stinchfield Award: identifying who will fail following irrigation and debridement for prosthetic joint infection: a machine learning-based validated tool. Bone Jt J. 2020;102(7):11-9.

59. Shah AA, Devana SK, Lee C, Kianian R, van der Schaar M, SooHoo NF. Development of a novel, potentially universal machine learning algorithm for prediction of complications after total hip arthroplasty. J Arthroplasty. 2021;36(5):1655-1662.e1.
60. Small SR, Bullock GS, Khalid S, Barker K, Trivella M, Price AJ. Current clinical utilisation of wearable motion sensors for the assessment of outcome following knee arthroplasty: a scoping review. BMJ Open. 2019;9(12):e033832.

61. Bahadori S, Immins T, Wainwright TW. A review of wearable motion tracking systems used in rehabilitation following hip and knee replacement. J Rehabil Assist Technol Eng. 2018:5:205566831877181.

62. Ramkumar PN, Haeberle HS, Ramanathan D, Cantrell WA, Navarro SM, Mont MA, et al. Remote patient monitoring using mobile health for total knee arthroplasty: validation of a wearable and machine learning-based surveillance platform. J Arthroplasty. 2019;34(10):2253-9.

63. Polus JS, Bloomfield RA, Vasarhelyi EM, Lanting BA, Teeter MG. Machine learning predicts the fall risk of total hip arthroplasty patients based on wearable sensor instrumented performance tests. J Arthroplasty. 2021;36(2):573-8.

64. Rouzrokh P, Wyles CC, Philbrick KA, Ramazanian T, Weston AD, Cai JC, et al. A deep learning tool for automated radiographic measurement of acetabular component inclination and version after total hip arthroplasty. J Arthroplasty. 2021;36(7):2510-2517.e6.

65. Jacofsky DJ, Allen M. Robotics in arthroplasty: a comprehensive review. J Arthroplasty. 2016;31(10):2353-63.

66. Thai MT, Phan PT, Hoang TT, Wong S, Lovell NH, Do TN. Advanced intelligent systems for surgical robotics. Adv Intell Syst. 2020;2(8):1900138.

67. Chand M, Ramachandran N, Stoyanov D, Lovat L. Robotics, artificial intelligence and distributed ledgers in surgery: data is key! Tech Coloproctol. 2018;22:645-8. Italy.

68. Zhou X-Y, Guo Y, Shen M, Yang G-Z. Application of artificial intelligence in surgery. Front Med. 2020;14(4):417-30

69. Panesar S, Cagle Y, Chander D, Morey J, Fernandez-Miranda J, Kliot M. Artificial intelligence and the future of surgical robotics. Ann Surg. 2019;270(2):223-6.

70. Li Z, Zhang X, Ding L, Du K, Yan J, Chan MTV, et al. Deep learning approach for guiding three-dimensional computed tomography reconstruction of lower limbs for robotically-assisted total knee arthroplasty. Int J Med Robot. 2021;e2300. https://doi.org/10.1002/rcs.2300.

71. Haeberle HS, Helm JM, Navarro SM, Karnuta JM, Schaffer JL, Callaghan $J J$, et al. Artificial intelligence and machine learning in lower extremity arthroplasty: a review. J Arthroplasty. 2019;34(10):2201-3.

72. Bini SA. Artificial intelligence, machine learning, deep learning, and cognitive computing: what do these terms mean and how will they impact health care? J Arthroplasty. 2018;33(8):2358-61.

73. Jayakumar P, Bozic KJ. Advanced decision-making using patient-reported outcome measures in total joint replacement. J Orthop Res Off Publ Orthop Res Soc. 2020;38(7):1414-22.

74. Jayakumar P, Moore MG, Furlough KA, Uhler LM, Andrawis JP, Koenig KM, et al. Comparison of an artificial intelligence-enabled patient decision aid vs educational material on decision quality, shared decision-making, patient experience, and functional outcomes in adults with knee osteoarthritis: a randomized clinical trial. JAMA Netw Open. 2021;4(2):1-14.

75. Safdar NM, Banja JD, Meltzer CC. Ethical considerations in artificial intelligence. Eur J Radiol. 2020;122:108768. Elsevier Ireland Ltd.

76. Gerke S, Minssen T, Cohen G. Ethical and legal challenges of artificial intelligence-driven healthcare. Artif Intell Healthc. 2020: 295-336. https:// doi.org/10.1016/B978-0-12-818438-7.00012-5. Elsevier.

77. Kelly CJ, Karthikesalingam A, Suleyman M, Corrado G, King D. Key challenges for delivering clinical impact with artificial intelligence. BMC Med. 2019;17(1):1-9.

78. Rodrigues R. Legal and human rights issues of Al: gaps, challenges, and vulnerabilities. J Responsible Technol. 2020;4:100005.

79. Morley J, Machado CCV, Burr C, Cowls J, Joshi I, Taddeo M, et al. The ethics of Al in health care: a mapping review. Soc Sci Med. 2020;260:113172. Elsevier Ltd.

80. Rigby MJ. Ethical dimensions of using artificial intelligence in health care. AMA J Ethics. 2019;21(2):121-4.

\section{Publisher's Note}

Springer Nature remains neutral with regard to jurisdictional claims in published maps and institutional affiliations. 